\title{
Rhythm of music seen through dance: Probing music-dance coupling by audiovisual meter perception
}

\author{
Yi-Huang Su${ }^{1}$ \\ ${ }^{1}$ Technical University of Munich, Munich, Germany
}

\author{
Correspondence: \\ Yi-Huang Su \\ Department of Movement Science, \\ Faculty of Sport and Health Sciences, \\ Technical University of Munich \\ Georg-Brauche-Ring 62, \\ 80992 Munich, Germany \\ Tel: +49-89-289-24552 \\ Email: yihuang.su@tum.de
}




\begin{abstract}
Action-perception coupling in music has been evidenced not only by how patterns of human dance reflect the metrical structure of musical rhythm, but also that moving to music modulates rhythm perception. Given the inherent connection between music and dance, this research investigated whether dance observation could induce meter perception of the visual rhythm similarly to the musical counterpart, and whether the visual meter could modulate concurrent auditory metrical perception. In Experiment 1, participants watched a point-light figure dance to a rhythm they heard simultaneously, both of which varied in meter. Participants responded whether the dance matched the rhythm, and the results were consistent with the imposed audiovisual meter match / mismatch, suggesting that participants could extract the visual meter from dance and compare it to the auditory meter. In Experiment 2, participants watched the dance in different meters while listening to a metrically ambiguous rhythm, which they (to some extent) subsequently identified as being more similar to another rhythm accentuated in the same meter as the dance than one in a different meter. The data partially supported visual modulation of auditory metrical interpretation. Together these results demonstrate parallels in meter perception between music and dance, which may share a common action representation that mediates cross-modal interactions. The findings also support theories of embodied musical rhythm from the perspective of visual-motor simulation.
\end{abstract}

Keywords: rhythm perception, music, dance, audiovisual, action perception, meter, embodied cognition 


\section{$1 \quad$ Introduction}

Moving spontaneously to music is a phenomenon most people can identify with. One musical component that especially engages human movement is its rhythm, which refers to the temporal patterns composed of hierarchical levels of periodicity (Cooper \& Meyer, 1963). Notably, humans also often move in a periodic manner to musical rhythm, and this tendency can be found in movements of different body parts (Burger, Thompson, Luck, Saarikallio, \& Toiviainen, 2014; Su \& Pöppel, 2012; Toiviainen, Luck, \& Thompson, 2010). It has emerged that periodic movements in the vertical and in the lateral dimensions tend to be synchronized to a faster and a slower musical pulse, respectively, and these movements can be present in parallel as are the different pulse levels in music (Burger et al., 2014; Toiviainen et al., 2010). In a now classic framework first published about a decade ago, Leman (2008) conceptualized this link between human movement and music as a manifestation of embodied music cognition: The movement pattern in response to music could reflect, or correspond to, the metrical structure of musical rhythm.

That human movements can embody musical rhythm is often taken as evidence of sensorimotor coupling in rhythm perception (Maes, Leman, Palmer, \& Wanderley, 2014), which is consistent with the common coding theory for action and perception (Prinz, 1997). According to this theory, sensory and motor systems are subserved by (at least partially) overlapping representations and neural mechanisms, and the interaction can be bidirectional. As such, action (i.e., moving in a certain pattern to music) is not merely consequent upon perception (i.e., hearing the pattern of musical rhythm), but may indeed influence the latter. Studies have shown that moving periodically along with an auditory rhythm can improve perception of a regular pulse (Su \& Pöppel, 2012) and of event timing (Manning \& Schutz, 2013). Moreover, bouncing whole-bodily in a given meter along with a metrically ambiguous auditory rhythm can lead the rhythm to be perceived in that meter (Phillips-Silver \& Trainor, 2005; 2007). Besides overt movements, musical rhythm also engages the internal motor system during passive listening (Repp \& Su, 2013), often shown as activations in the cortical and subcortical motor areas in rhythm tasks that do not require relevant motor output (Grahn, 2012; Stupacher, Hove, Novembre, Schütz-Bosbach, \& Keller, 2013). In terms of internal motor influence on perception, the Action Simulation for Auditory Prediction (ASAP) hypothesis (Patel \& Iversen, 2014) proposed that the motor system may simulate periodic body movement covertly (without actually moving along) upon hearing a musical rhythm. Such motor activities would generate predictive signals that are communicated to the auditory system online, which is instrumental to beat perception. Taken together, the coupling between rhythm and movement -in both covert and overt forms - seems fairly convincing.

There is, however, one missing link in this picture. Overt movements along with music - be it spontaneous body motion (Burger et al., 2014) or choreographed dance (Naveda \& Leman, 2010) are relatively straightforward and measureable, and so are their effects on rhythm perception (Manning \& Schutz, 2013; Phillips-Silver \& Trainor, 2007; Su \& Pöppel, 2012). Covert movements, by contrast, could be generated in different ways, the effect of which on rhythm perception has not been disambiguated. According to the motor simulation theories (Jeannerod, 2001; Vogt, Di Rienzo, Collet, Collins, \& Guillot, 2013), movements can be simulated by action observation or by action imagery, both of which have been demonstrated using music-related movements such as dance (Kirsch \& Cross, 2015). While the ASAP hypothesis suggests that the internal simulation supporting beat perception occurs at an abstract level without necessarily involving motor imagery (Patel \& Iversen, 2014), it remains an intriguing question whether and how other forms of motor simulation may evoke rhythm representation. Two recent studies (Su, 2016a; 2016b) show that, when watching (in silence) point-light dance movements that embody a specific metrical structure (e.g., a 4/4 
musical meter), observers perceive the dance rhythm visually using similar sensorimotor mechanisms to those for the auditory musical rhythm. Besides, moving visual stimuli - compared to static ones are found to be more effective in communicating the visual rhythm (Grahn, 2012; Iversen et al., 2015), in competing with the auditory rhythm as pacing signals (Hove, Iversen, Zhang, \& Repp, 2013a), and in activating the motor system (Hove, Fairhurst, Kotz, \& Keller, 2013b). These findings point to the possibility that observing dance movements may indeed lead to internal action simulation of the movement rhythm in ways similar to what has been proposed for the auditory counterpart. As such, it seems reasonable to ask whether the motor system can respond to the rhythm of an observed dance movement along with music. Can the sensorimotor system in this case simulate movement patterns beyond a single periodicity to represent, for example, the meter? What are the effects of dance observation on musical rhythm perception, given possible motor simulation for both music and dance?

The present research addressed these issues using a bimodal paradigm, in which participants watched a point-light figure (PLF, see Johansson, 1973) dancing to a percussive rhythm they heard at the same time. The scenario resembled being a spectator in a dance performance, where both streams of (visual) dance and (auditory) music information were simultaneously available (Jola et al., 2013). The movements were derived from the basic step of the Charleston dance and were taken from two recent studies ( $\mathrm{Su}, 2016 \mathrm{a} ; 2016 \mathrm{~b})$. The Charleston dance is typically performed along with swing music of a 4/4 meter, and unsurprisingly the movement appears to embody this metrical structure (Naveda \& Leman, 2010): Within one movement cycle corresponding to eight pulses, the trunk bounces vertically at every pulse while the legs move laterally at every second pulse, and the spatiotemporal pattern of the leg movements can be grouped in four (i.e., two kicks of the left leg and two of the right; see Su, 2016b for detailed movement descriptions) ${ }^{1}$. Su (2016a; 2016b) established that observers perceive the two metrical levels accordingly from the movement pattern, namely the periodicity of the trunk and that of the legs, with the latter marking the visual beat. The studies also show that the two levels are perceived in parallel as beat (leg movement) and metrical subdivisions (trunk movement), mirroring the well-known auditory mechanism. Following these findings, the present study further hypothesized that this dance movement as a whole could communicate a visual rhythm, in which the leg movement pattern could give rise to the visual meter. Accordingly, observing this dance should induce beat perception in that meter by means of motor simulation, which may be comparable to its auditory counterpart and may even modulate auditory metrical perception.

Two experiments were conducted to address different questions with respect to the hypothesis formulated above. The first experiment investigated whether, when watching a dance movement and listening to a rhythm simultaneously, participants could perceive the visual meter of the dance and judge its compatibility to the auditory meter. The Charleston basic step was performed either in its original duple-meter pattern (four leg kicks: left - left - right - right), or in a modified triple-meter pattern (three leg kicks: left - left - right, whereby one movement cycle corresponded to six instead of eight pulses). The auditory rhythm was similar to those used by Phillips-Silver and Trainor (2007), which was either metrically ambiguous, accentuated in a duple meter, or accentuated in a triple meter. Participants were instructed to answer intuitively on each trial whether the pattern of the dance matched that of the auditory rhythm. If the visual dance meter emerged perceptually in a similar manner as the auditory musical meter, there should be more "match" responses in conditions with the

\footnotetext{
${ }^{1}$ Note that this dance also has the advantage of encompassing movement patterns that match how untrained individuals move different body parts spontaneously to musical rhythms (Toiviainen et al., 2010).
} 
same auditory and visual meter (by stimulus design) than in those with mismatching ones. In conditions with an ambiguous auditory rhythm, the match response for either visual meter should be similar.

Using the same auditory and visual stimuli, the second experiment examined the effect of the visual meter on auditory metrical perception in a task similar to that adopted by Phillips-Silver \& Trainor (2007). The question was whether observing the dance performed in a duple or triple meter while listening to a rhythm could lead the rhythm to be interpreted in that meter accordingly. Participants listened to a metrically ambiguous rhythm while watching the dance in either meter, after which they listened to two different metrically accentuated rhythms (one in each meter) without any visual stimuli. The task was to judge which of the two accented rhythms was more similar to the first, ambiguous one. If the visual meter induced by dance observation influenced concurrent auditory metrical perception, there should be a higher percentage of choosing the auditory rhythm accentuated in the same meter as the visual dance than in a different meter.

\section{Experiment 1: Metrical match between auditory and visual rhythms}

\subsection{Methods}

\subsubsection{Participants}

Twenty-one young, healthy volunteers (five males, mean age 23.7 years, $\mathrm{SD}=3.8$ ) took part in this experiment. Participants were naïve of the purpose, gave written informed consent prior to the experiment, and received an honorarium of $8 €$ per hour in return. Eighteen and twelve participants had trained in music and dance (none in swing dance) as amateurs, respectively, amongst whom ten had learned both. The mean training duration of music and dance was 6.3 years $(\mathrm{SD}=4.3)$ and 4.7 years $(\mathrm{SD}=5.7)$. The study had been approved by the ethic commission of Technical University of Munich, and was conducted in accordance with the ethical standards of the 1964 Declaration of Helsinki.

\subsubsection{Stimuli and materials}

\subsubsection{Visual Stimuli}

The visual stimuli consisted of a human PLF performing the basic steps of the Charleston dance in two different tempi. The stimuli had been generated by recording a swing dancer performing these steps using a 3-D motion capture system (Qualisys Oqus, 8 cameras at a sampling rate of $200 \mathrm{~Hz}$, with 13 markers attached to the joints, [Johansson, 1973]), paced by metronomes with an inter-onset interval (IOI) of 400 and $450 \mathrm{~ms}$, respectively. Detailed descriptions of stimuli preparation and construction can be found in a recent study ( $\mathrm{Su}, 2016 \mathrm{~b})$, from which the stimuli were taken.

The dance was performed in continuous cycles, with one cycle corresponding temporally to eight metronome beats. Within a cycle, the trunk bounced vertically at every beat (beat 1 to 8 ) while the legs moved laterally at every second beat (left leg kicking back at beat 1 and forth at beat 3; right leg kicking forth at beat 5 and back at beat 7). The same cycle was looped. In the original recording the arms also swung laterally at every second beat, as was authentic of the Charleston dance. In the present stimuli, in order to focus on the metrical accents communicated by the leg movements, the arm movements were removed by adjusting the trajectories of the elbow and hand markers, such that the hands were placed upon the hips throughout the cycle. (See Su, 2016a for the same manipulation, which also showed that the absence of arm movements did not alter rhythm perception of the leg movements). Fig. 1, $1^{\text {st }}$ panel. 
Two metrical versions of the dance were created. One was derived from the original Charleston that, as described above, lasted eight IOIs per cycle and encompassed eight vertical trunk bounces and four lateral leg movements (two of the left and two of the right). This version fit temporally in a duple (4/4) musical meter (Fig. 1, $1^{\text {st }}$ panel. See Supplementary Material Video 1). The other version was modified such that one dance cycle corresponded to six IOIs, encompassing six trunk bounces and three leg movements (two of the left and one of the right). Namely, the last kick of the right leg in the original version was skipped ${ }^{2}$. This version fit temporally in a triple (3/4) musical meter (Fig. 1, $2^{\text {nd }}$ panel. See Supplementary Material Video 2).

The 3-D motion data of each dance were presented as point-light display on a 2-D monitor, using routines of Psychophysics Toolbox version 3 (Brainard, 1997) running on Matlab ${ }^{\circledR}$ R2012b (Mathworks). The PLF was represented by 13 white discs against a black background, each of which subtended 0.4 degrees of visual angle $\left(^{\circ}\right)$. The whole PLF subtended approximately $5^{\circ}$ (width) and $12^{\circ}$ (height) when viewed at $80 \mathrm{~cm}$. The PLF was displayed facing the observers, in a configuration as if the observers were watching from $20^{\circ}$ to the left of the PLF, which served to optimize depth perception of biological motion in a 2-D environment (using the moglDrawDots $3 D$ function in the toolbox).

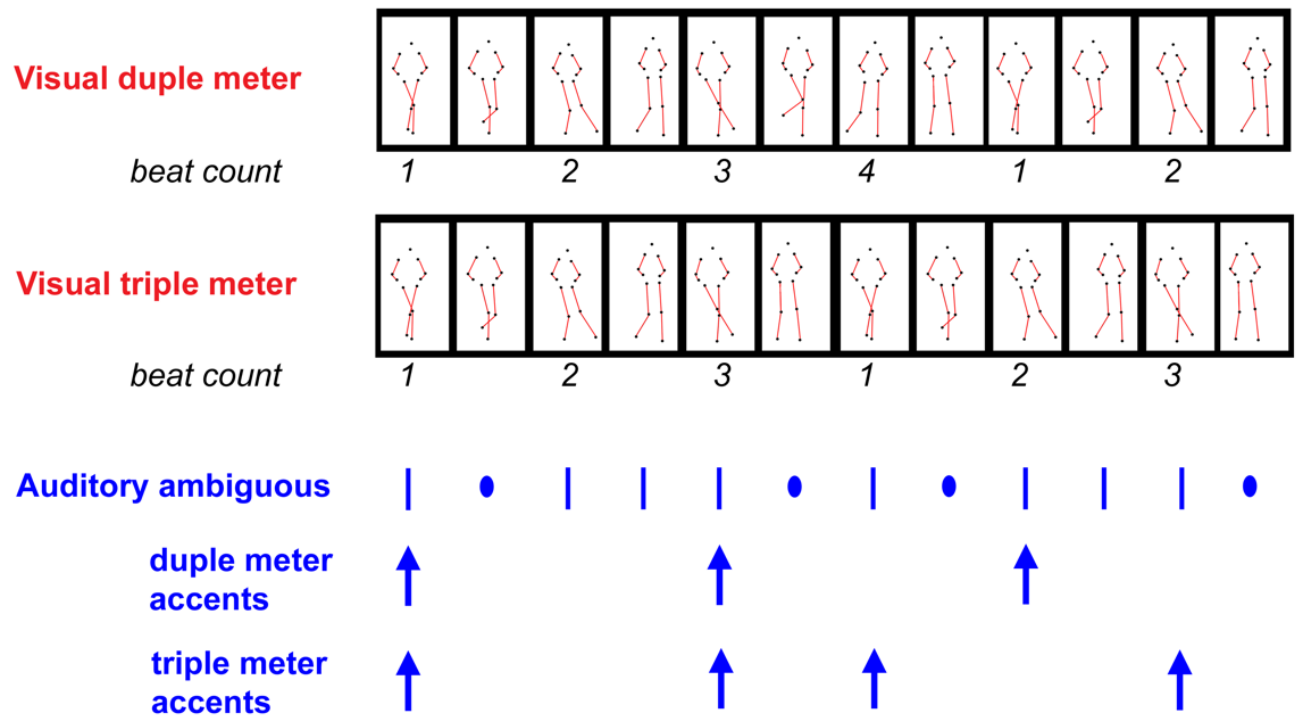

Figure 1. Auditory and visual stimuli in Experiment 1. Participants watched a PLF dancing either in a duple meter $\left(1^{\text {st }}\right.$ panel $)$ or in a triple meter $\left(2^{\text {nd }}\right.$ panel $)$ to an auditory rhythm that was one of the three: metrically ambiguous $\left(3^{\text {rd }}\right.$ panel), accented in a duple meter $\left(3^{\text {rd }}\right.$ and $4^{\text {th }}$ panels $)$, or accented in a triple meter $\left(3^{\text {rd }}\right.$ and $5^{\text {th }}$ panel). The task was to judge whether the pattern of the auditory rhythm and that of the dance matched. Numbers below each visual dance sequence indicate the beat count derived from the leg movement pattern. Vertical lines and ovals in the ambiguous auditory rhythm depict discrete events of snare drum and high-hat sounds, respectively. Metrically accented rhythms consisted of the metrically ambiguous one with additional accents of a bass drum sound depicted by the arrows. Both the auditory and visual rhythms presented in the experiment lasted twice as long as depicted here (i.e., 24 IOIs in total).

\footnotetext{
2 This version had also been generated by recording the dancer performing the modified steps, so that the movement was as naturalistic as the original version.
} 


\subsubsection{Auditory Stimuli}

The auditory stimuli were rhythmic patterns, each consisting of successive discrete events. Three types of rhythm were generated based on their metrical accentuation: (1) metrically ambiguous, (2) accented in a duple meter, and (3) accented in a triple meter. Each rhythm type was created in two different tempi as for the visual stimuli. The sounds were generated by the music software Logic 8 Express (Apple Inc. California).

The metrically ambiguous rhythms were composed in the same manner as described in PhillipsSilver and Trainor (2007): One rhythm consisted of six isochronous events played by two different percussion instruments alternatingly. The rhythm had a pattern of $\mathrm{A}-\mathrm{B}-\mathrm{A}-\mathrm{A}-\mathrm{A}-\mathrm{B}$, where $\mathrm{A}$ and $\mathrm{B}$ here were played by a snare drum and a high-hat, respectively. This rhythm could not be consistently heard in groups of three or four, thus not yielding clear metrical accents in a triple or duple meter (Fig. 1, $3^{\text {rd }}$ panel, depicting two cycles of the rhythm. See Supplementary Material Audio 1). In order to avoid the rhythm being perceptually segmented in the same way across trials, two more variations of this rhythm were generated based on the starting phase. In one, the rhythm started with the third event, and the first two events were placed in the end (i.e., A-A-A-B-A-B); in the other, the rhythm started with the fifth event, with the first four events placed in the end (i.e., A-B-A-B-A-A). For each trial in the experiment, four continuous cycles of the same rhythm were presented, encompassing twenty-four events and lasting twenty-four IOIs.

The two types of metrically accented rhythms were first generated in the same manner as the metrically ambiguous ones, with also three variations of a rhythm. On top of that, an accent played by a bass drum sound was added in parallel to the rhythm: For the duple-meter rhythms, the bass drum coincided with every fourth event when two cycles of the six-event rhythm were concatenated, i.e., the $1^{\text {st }}, 5^{\text {th }}$, and $9^{\text {th }}$ events (See Supplementary Material Audio 2). For the triple-meter rhythms, the bass drum coincided with the $1^{\text {st }}, 5^{\text {th }}, 7^{\text {th }}$, and $11^{\text {th }}$ events (Fig. 1, lowest three panels. See Supplementary Material Audio 3). As such, the duple-meter rhythms could be grouped in fours or eights, and the triple-meter rhythms could be grouped in sixes. The metrical grouping was based on the recurrent pattern of the bass drum accents paired with the rhythm tones, in which the oddnumbered positions represented the main beat level and the even-numbered positions represented the subdivisions. Note that both the duple- and the triple-meter rhythms were accentuated such that, when presented together with the dance in the same meter, the bass drum accents always coincided with the left back-kick (beat 1) and the right front-kick (beat 3) of the legs (beat number as labeled for the visual stimuli in Fig. 1). Accents on beat 2 and (in the case of the duple-meter rhythm) beat 4 positions were omitted so as to avoid possible strategies of matching the auditory and visual rhythms solely based on co-occurring accents and kicks without regarding their metrical grouping.

\subsubsection{Procedure and design}

The stimuli and experimental program were controlled by a customized Matlab script and Psychtoolbox version 3 routines running on a Mac OSX environment. The visual stimuli were displayed on a 17-inch CRT monitor (Fujitsu X178 P117A) with a frame frequency of $100 \mathrm{~Hz}$ at a spatial resolution of $1024 \times 768$ pixels. Participants sat with a viewing distance of $80 \mathrm{~cm}$. Sounds were delivered by closed studio headphones (AKG K271 MKII) at a comfortable level that was fixed across participants. 
On each trial, participants watched a PLF dancing the Charleston step to an auditory rhythm they heard at the same time, at one of the two tempi (IOI $=400$ or $450 \mathrm{~ms}$ ). The auditory and the visual tempi were always the same for a given trial. The dance was performed either in the duple or the triple meter. The auditory rhythms could be one of the three metrical types: metrically ambiguous, duple meter, or triple meter (Fig. 1). For each trial, the auditory rhythm and the dance movement both lasted 24 IOIs. Participants were instructed to watch the dance and listen to the rhythm simultaneously, following which they were required to judge whether the dance movement matched the auditory rhythm, in a Yes / No forced choice task. Participants were instructed to base their judgment subjectively and intuitively on whether the movement pattern fit the pattern of the rhythm. Participants were explained that the movement and rhythm were of the same tempo and were synchronous, on which the judgment should not be based. They were also informed that there was no correct or incorrect answer. No further restriction was given.

The experiment followed a 2 (visual meter) $\times 3$ (auditory meter) $\times 2$ (tempo) design, each with 12 repetitions. The three phase-based variations of the auditory rhythm occurred with equal frequency for every experimental condition, which was not analyzed as a factor. The trials were presented in 6 blocks of 24 trials each, with all the conditions balanced across blocks and the order of conditions randomized within a block. Participants practiced 5 trials before starting the experiment. The experiment was completed in around 1 hour.

\subsection{Results}

For each participant, the percentage of responding "match" was calculated for each of the experimental conditions. The individual percentages were submitted to a 2 (visual meter) $\times 3$ (auditory meter) $\times 2$ (tempo) repeated-measures ANOVA. Greenhouse-Geisser correction was applied to the $\mathrm{p}$ values of effects of auditory meter. Tukey HSD test was used for post-hoc comparisons. For each critical effect identified by the ANOVA, the estimated Bayes factor in favor of the effect (Rouder, Morey, Speckman, \& Province, 2012) was also supplemented to indicate its strength.

There was a main effect of visual meter, $F(1,20)=45.79, p<0.001, \eta_{p}{ }^{2}=0.70$ (Bayes factor $1.2 \times$ $1018: 1$ in favor of the effect), showing a higher percentage of match response for the visual triple meter than for the visual duple meter. The main effect of auditory meter was also significant, $F(2,40)$ $=4.91, p=0.02, \eta_{p}{ }^{2}=0.20$ (Bayes factor $2.9 \times 106: 1$ ), and the post-hoc comparisons revealed a lower percentage of match response for the duple meter than for the ambiguous $(p=0.025)$ or the triple meter condition ( $p=0.006)$, while the latter two did not differ from each other. There was also an effect of tempo, $F(1,20)=18.02, p<0.001, \eta_{p}{ }^{2}=0.47$ (Bayes factor $55041: 1$ ), with a higher percentage of match response for the faster tempo (IOI $=400 \mathrm{~ms})$. See Fig. 2a.

There was a significant interaction between the auditory and visual meters, $F(2,40)=11.71, p<$ $0.001, \eta_{p}{ }^{2}=0.37$ (Bayes factor $4.0 \times 105: 1$ in favor of the interaction). Following that, partial oneway ANOVAs were carried out for each auditory meter separately: For both the auditory ambiguous and the auditory triple meter conditions, visual triple meter yielded higher percentages of match response than visual duple meter, $F(1,20)=20.34, p<0.001, \eta_{p}{ }^{2}=0.50$, and $F(1,20)=47.00, p<$ $0.001, \eta_{p}{ }^{2}=0.70$. However, for the auditory duple meter conditions, there was no difference between visual duple and visual triple meters, $F(1,20)=0.25, p=0.63, \eta_{p}{ }^{2}=0.01$. The $95 \% \mathrm{CI}$ of the difference scores (Masson \& Loftus, 2003) between the two visual meters for each auditory condition reveals that, across tempi, the visual effect was most consistent (i.e., difference greater than zero at the $95 \% \mathrm{CI}$ ) in the auditory triple meter condition (Fig. 2b). 
As an alternative way of contrasting, partial one-way ANOVAs were also carried out for each visual meter separately: For the visual duple meter, the auditory meter did not influence response, $F(2,40)$ $=1.08, p=0.35, \eta_{p}{ }^{2}=0.05$. For the visual triple meter, the auditory meter had an effect, $F(2,40)=$ $17.84, p<0.001, \eta_{p}{ }^{2}=0.47$, and post-hoc comparisons showed more match response in the auditory triple meter and auditory ambiguous than in the auditory duple meter conditions, both $p \mathrm{~s}<0.001$, while the former two did not differ from each other. No other significant interactions were found.

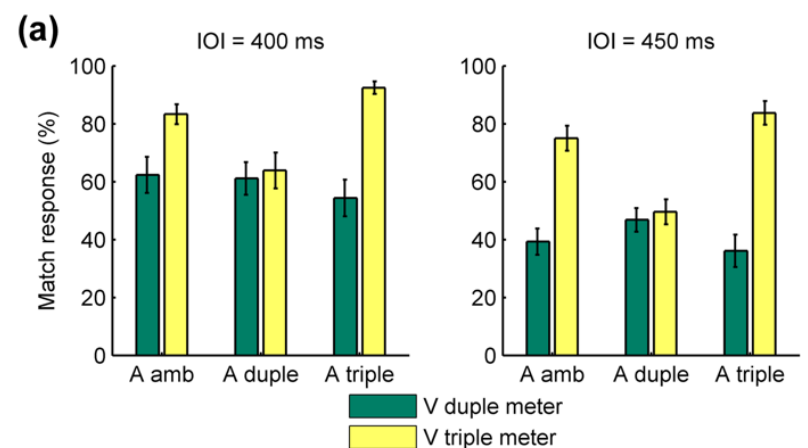

(b)

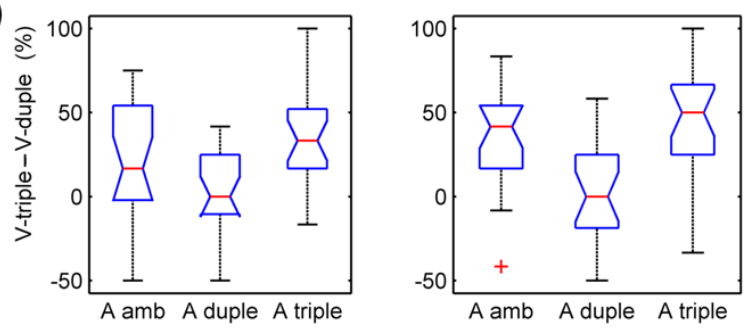

Figure 2. Results of Experiment 1. (a) Mean percentages of "match" response for each visual meter condition as a function of the auditory meter, for each audiovisual tempo separately. A amb, A duple, and $A$ triple on the $X$-axis represent auditory conditions of metrically ambiguous, duple meter, and triple meter. Error bars represent standard error of the means. (b) Effects of visual meter contrasted at each auditory meter condition, showing the median difference scores across participants (red horizontal lines) and their $95 \%$ CIs (the blue notches). The whiskers represent $99 \%$ of the data distribution.

In sum, the visual dance movement in a triple meter, compared to that in a duple one, was overall judged to be more fitting to the auditory rhythms regardless of the auditory meter. This pattern was also evident when the auditory rhythm was metrically ambiguous. Given this baseline preference, the effect of visual meter was still shown when the auditory rhythm had a triple meter (more match response for visual triple than for visual duple meter), while this preference disappeared when the auditory rhythm had a duple meter. Similarly, when the PLF danced in a triple meter, the percentage of match response dropped for auditory rhythms accentuated in a duple meter compared to the other auditory conditions.

\section{Experiment 2: Visual influence on auditory metrical interpretation}

Using a similar paradigm to Phillips-Silver and Trainor (2007, Experiment 4) with a few modifications, this experiment examined whether a metrically ambiguous auditory rhythm would be 
more often interpreted in a duple or triple meter if the rhythm was accompanied by a dance movement in that meter.

\subsection{Method}

\subsubsection{Participants}

Twenty-two volunteers (five males, mean age 26.8 years, $\mathrm{SD}=3.7$ ) participated. Sixteen and seventeen participants had learned musical instruments and dance (none in swing dance), respectively, amongst whom thirteen had learned both (all amateurs). The mean music and dance training duration was 4.3 years $(\mathrm{SD}=3.8)$ and 5 years $(\mathrm{SD}=5.2)$, respectively. The participant handling and ethics procedure was the same as in Experiment 1.

\subsubsection{Stimuli and materials}

The visual and auditory stimuli were the same as used in Experiment 1.

\subsubsection{Procedure and design}

The setup was the same as in Experiment 1. On each trial, participants watched a PLF dancing the Charleston basic steps to an auditory rhythm they heard at the same time, at one of the two tempi. The dance was performed either in a duple or in a triple meter. The auditory rhythm, termed the "standard rhythm", was always metrically ambiguous. Both the auditory and the visual sequences lasted 24 IOIs. Immediately following that, participants heard two different rhythms consecutively without visual stimuli, termed "comparison rhythms". One of the comparison rhythms was accentuated by the bass drum in a duple meter, and the other in a triple meter, the order of which was random but equally frequent across the experiment. Both comparison rhythms lasted 12 IOIs. Participants were instructed to watch the PLF move to the standard rhythm; following the two comparison rhythms, they were required to answer which of the two was more similar to the standard one in a 2AFC task (Fig. 3). Note that for each trial, the three auditory rhythms (one standard followed by two comparisons) consisted of the three phased-based variations, the order of which was random but balanced across all conditions. As such, there were never any identical rhythms amongst the standard and comparison stimuli within a trial. Participants were informed that all three rhythms were non-identical, and the judgment should be based subjectively and intuitively on which comparison rhythm had a more similar pattern to the standard one. This experiment followed a 2 (visual meter) $\times 2$ (tempo) design, presented in 4 blocks of 12 trials each, with all the conditions balanced across blocks and the order of conditions randomized within a block. Participants practiced 5 trials before starting the experiment, which was completed in around 45 minutes. 


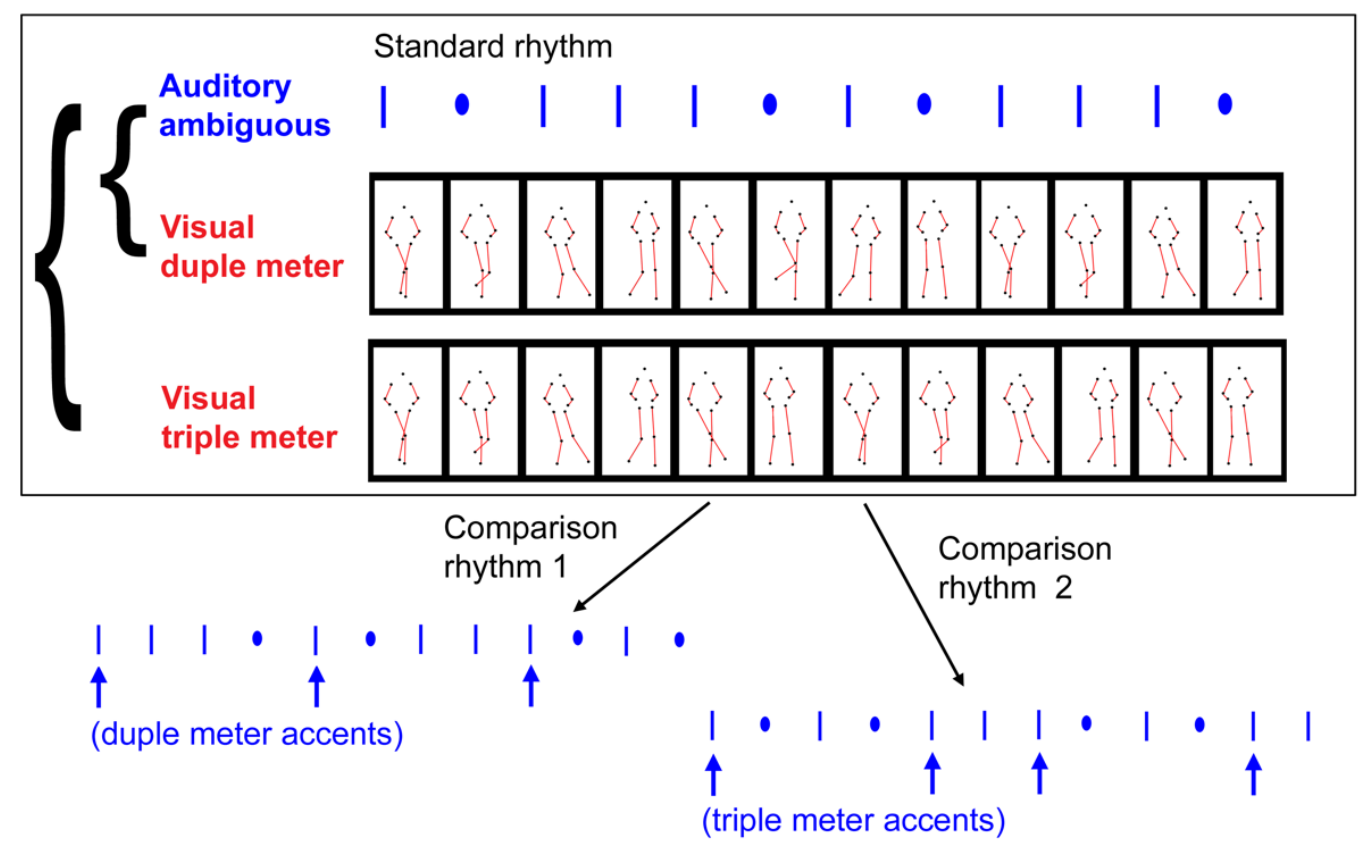

Question: Which comparison rhythm is more similar to the standard rhythm?

Figure 3. Stimuli and procedure in Experiment 2. Participants listened to a metrically ambiguous rhythm ( $1^{\text {st }}$ panel, termed "standard rhythm") while watching a PLF dance either in a duple meter $\left(2^{\text {nd }}\right.$ panel) or in a triple meter ( $3^{\text {rd }}$ panel) to this rhythm. Following that, they heard two comparison rhythms in succession, one of which was accented in a duple meter (lower left panel) and the other in a triple meter (lower right panel). The task was to judge which of the two comparisons was more similar to the standard rhythm. The standard rhythm and the accompanying visual dance presented in the experiment lasted twice as long as depicted here (i.e., 24 IOIs).

\subsection{Results}

Data from one participant were excluded from analyses, as post-experiment interview revealed that the participant had failed to comprehend the task. The total sample size was thus twenty-one. The data were analyzed in the same way as described in Phillips-Silver and Trainor (2007): For each participant, the percentage of choosing the auditory duple meter was calculated for each of the experimental conditions. The individual percentages were submitted to a 2 (visual meter) $\times 2$ (tempo) repeated-measures ANOVA, which identified a main effect of visual meter, $F(1,20)=5.23, p=0.03$, $\eta_{p}{ }^{2}=0.20$ (Bayes factor 39:1 in favor of the effect), showing a higher percentage of choosing the auditory duple meter when the visual dance was in a duple than in a triple meter (Fig. 4). No other effect or interaction was found. Thus, across both tempi, participants more often judged the ambiguous auditory rhythm to be similar to the variation in a duple meter when the ambiguous rhythm had been accompanied by the duple-meter (compared to the triple-meter) dance. Note that while the data in Fig. 4 show the mean percentages of choosing the auditory duple meter, the mean percentages of choosing the auditory triple meter could be derived by subtracting each mean here from $100 \%$ (i.e., the length of the rest of each bar until 100\%). In this perspective, participants more often judged the ambiguous rhythm to be similar to the triple-meter variation when it had been accompanied by the triple-meter (compared to duple-meter) dance. 


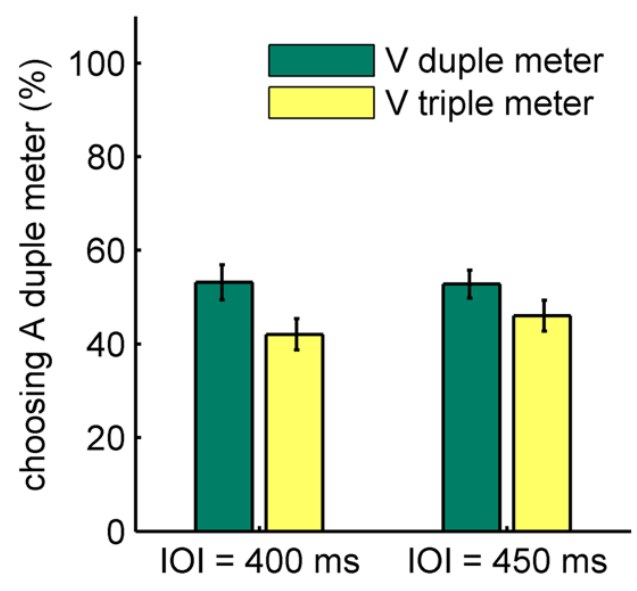

Figure 4. Results of Experiment 2. Mean percentages of choosing the auditory duple-meter rhythms for each visual meter condition, at each audiovisual tempo. Error bars represent standard error of the means.

The results above demonstrated the difference in auditory metrical interpretation between visual conditions, indicating a relative visual effect on auditory interpretation. When comparing between individual percentages of choosing the auditory duple meter and choosing the auditory triple meter under each visual meter condition, though, a significant difference was only found for the visual triple meter at the faster tempo (IOI $=400 \mathrm{~ms}), t(20)=2.39, p=0.027$, confirming a greater percentage of choosing the auditory triple meter than choosing the auditory duple meter. For the other visual conditions, however, the differences were not statistically significant. As such, the current results only partially supported an absolute effect of the visual meter on biasing auditory metrical perception.

\section{Discussion}

Motivated by theories of musical embodiment (Leman, 2008) and internal action simulation for auditory rhythm perception (Patel \& Iversen, 2014), the present study investigated whether rhythm representations involving beat and meter could emerge covertly when observing human movements tightly coupled to music: dance, and how such visual action information interacted with musical rhythm in bimodal perception. Experiment 1 showed that participants were able to differentiate the metrical structure of the observed dance movement in relation to that of the rhythm they heard in parallel. This suggests that the visual dance rhythm, specifically its meter derived from the limb movement pattern, was perceived in a manner comparable to how the musical meter was perceived, i.e., involving accentuation and grouping of events. Experiment 2 offered partial support for the hypothesis that the visual meter could modulate metrical perception of a concurrent auditory rhythm, which seemed to be the case under a specific visual condition and when comparing the relative tendency in which auditory metrical interpretation was biased (i.e., comparison between visual meter conditions). While the present results do not argue for a strong visual influence on altering auditory meter perception, the findings show parallels in rhythm perception beyond a single periodicity between (auditory) music and (visual) dance (Su \& Salazar-Lopez, 2016), which may interact in perception at the level of meter depending on the tasks and conditions. 
The data pattern in Experiment 1, namely the interaction between the auditory and visual meter conditions, generally agreed with the hypothesized audio-visual metrical match, with a few unexpected results warranting discussions. First, the audio-visual match / mismatch was most consistently differentiated when the auditory rhythm was accentuated in a triple meter, in which the triple-meter dance yielded a higher percentage of "match" response than the duple-meter one, as predicted. This pattern was, however, not as clearly shown for the auditory rhythms accentuated in a duple meter. The result may be explained together with the observation that participants also more often matched the ambiguous auditory rhythms with the triple-meter dance, which contradicted the expectation of neutral preference. It would seem as if the present auditory rhythms, prior to additional accentuation, were preferentially matched with a triple-meter dance due to the fact that the auditory pattern repeated every six events. Thus, the segmentation of the rhythm corresponded better to that of the triple-meter dance (one cycle lasting six pulses) than to the duple-meter one (one cycle lasting eight pulses), which led to a pre-existing bias in the audio-visual matching. The main effect of visual meter also supported this speculation. As such, while adding triple-meter accents to these auditory rhythms likely enhanced their structural similarity to the triple-meter dance, the segmentation bias may have substantially reduced the metrical saliency when the duple-meter accents were added. As a result, auditory rhythms accentuated in a duple meter could not be clearly matched to the duplemeter dance. Nevertheless, the data still favor the interpretation that participants based their judgments on the metrical grouping of the auditory and visual rhythms rather than on cycle segmentation alone, as the latter strategy would have led to the same response pattern in all the auditory conditions, which was not the case. The fact that the "match" percentage for the triple-meter dance dropped in the auditory duple-meter conditions could be seen as the auditory duple-meter accents being perceived as less compatible with the visual triple meter, suggesting that metrical accentuations in both the auditory and visual rhythms were at play.

Results of Experiment 2 suggest that, to some extent, metrical interpretation of an ambiguous auditory rhythm may be modulated by the meter of a simultaneously observed dance movement: The ambiguous auditory rhythm accompanied by a triple-meter dance was more often perceived to be similar to a triple-meter than to a duple-meter variation. This visual effect, however, was not obtained with the duple-meter dance movement, which may be due to the visually imposed duplemeter accents being weakened by the structure of the auditory rhythm that favored triple-meter segmentation, as discussed in the results of Experiment 1. In addition, the visual effect was only shown at the faster tempo (IOI $=400 \mathrm{~ms}$, or $150 \mathrm{BPM}$ ), suggesting that the visual accents in the slower tempo (IOI $=450 \mathrm{~ms}$ ) was less effective in this regard. This seems consistent with a few previous findings that the visual metrical structure of this dance movement, i.e., its beat and subdivisions, is most optimally perceived at the tempo corresponding to the IOI of $400 \mathrm{~ms}(\mathrm{Su}$, 2016a; 2016b).

With respect to the relative visual effect in Experiment 2, shown as the difference in choosing one auditory meter between the two visual meter conditions, the direction of the effect seems to agree with that found by Phillips-Silver and Trainor $(2005 ; 2007)$ through real movements. Namely, the ambiguous auditory rhythm was more likely to be perceived in a specific meter when it had been accompanied by a dance in that meter than by a dance in a different meter. In their studies, though, the effect of movement meter on auditory metrical interpretation was found only when participants bounced whole-bodily in a duple meter (bouncing to every second event) or in a triple meter (bouncing to every third event) along with the auditory rhythm, but not in a visual condition where participants passively observed the same movements performed by another individual. As such, it may seem less surprising that the present visual effects were not as strong as those obtained in their movement conditions, and that the absolute response differences between the present visual 
conditions were clearly smaller than those found between their movement conditions. This suggests that overt actions are still more effective in modulating auditory temporal processing (Morillon, Hackett, Kajikawa, \& Schroeder, 2015) than covert ones induced by action observation, and that other mechanisms connected to real body movement may also be instrumental, such as the integration of vestibular signals during natural movement (Phillips-Silver \& Trainor, 2008).

While the present visual effects in Experiment 2 did not completely replicate the effects of actual movement in Phillips-Silver and Trainor (2005; 2007), it is worth noting - despite several similarities - how the present stimuli and paradigm differed from theirs, which may have influenced the results. Specifically, a few modifications were introduced in the present design that made the task more demanding: For one, Phillips-Silver and Trainor $(2005 ; 2007)$ presented as comparison rhythms the same auditory pattern as the standard (ambiguous) one, where accents were created by increasing the loudness of specific events. In the present task, by contrast, all three rhythms presented in a trial differed from one another by the starting phase, and the comparison rhythms were shorter than the standard one (see sections 2.1.2.2 and 3.1.3), which increased their overall dissimilarity and discouraged strategies such as memorizing the standard rhythm pattern and counting the accents. In this way, judgment of structural similarity would have to be implicitly based on higher-level metrical grouping rather than on matching of local, individual events. This approach was meant to ensure that the observed effect, if any, could indeed represent visual modulation on the meter level. It is likely that the stimuli as such made it more difficult for participants to extract similarity amongst different metrical variations, which lowered their tendency to choose one or the other comparison rhythm. In addition, whereas Phillips-Silver and Trainor implemented the two movement conditions between groups, the present experiment adopted a within-participant design, which was intended to minimize potential individual differences that might in any way be associated with different experimental conditions. This may have reinforced the pre-existing preference for pairing the ambiguous rhythm with the triple-meter dance (as already evidenced in Experiment 1), which led to ineffective accentuation of the duple-meter dance stimuli.

Given these considerations, the fact that the visual effect was still partially found - as opposed to the null effect in Phillips-Silver and Trainor's studies - points to the potential of the present dance stimuli to interact with auditory musical rhythms. It is possible that, in order to modulate musical meter perception, which is based on hierarchical modules such as pulse, beat, and metrical grouping (London, 2012), visual rhythms of comparable metrical complexity are required. Dance movements such as the present ones have the advantage of conveying visual metrical hierarchy naturalistically (Su, 2016a; 2016b) and possibly by engaging the internal motor system (Cross, Kraemer, Hamilton, Kelley, \& Grafton, 2009), yielding an effective visual counterpart to the auditory rhythm (Hove et al., 2013b). Besides, dance being tightly associated with music provides the optimal context compatibility to promote inter-sensory interactions (Doehrmann \& Naumer, 2008), whereby visual modulation of auditory beat perception has been previously shown ( $\mathrm{Su}, 2014)$. The present finding is also reminiscent of the well-known "McGurk effect" in audiovisual speech perception (McGurk \& MacDonald, 1976), in which the visual information of facial movements - also consisting of rhythmic temporal patterns (Ghazanfar \& Takahashi, 2014) - influences or even alters auditory perception.

As a whole, the present research built on two previous studies that systematically investigated perceptual and synchronization mechanisms with visual rhythms of the basic swing dance movements (Su, 2016a; 2016b). While these studies revealed several similar mechanisms for dance to those for music, they focused mainly on visual beat and tempo perception. The present study went further to examine a higher-level construct, meter, in dance rhythm by directly relating it to the 
auditory counterpart in a bimodal paradigm. In doing so it made a stronger and more ecological case for the music-dance comparison. Results from both experiments support the role of the leg movement pattern in communicating the visual meter. These mechanisms seem generalizable at least within a temporal range, where some tempi may be more optimal than others. Together with the previous findings, a more complete proposal for visual rhythm perception in dance - one that involves hierarchical modules as for musical rhythm - may be put forward: First, multiple periodicities can be perceived in parallel in different moving body parts, such as the trunk (lowerlevel pulse) and the limbs (higher-level pulse, i.e., the beat, especially from the leg movements, see $\mathrm{Su} \&$ Salazar-Lopez, 2016). On top of that, extracting the recurrent spatiotemporal pattern of the leg movements may be analogous to grouping the musical beat, giving rise to the percept of meter. One possible limitation at present is that, as visual meter perception in dance movement is a new field of investigation, it was necessarily probed by measuring the effect of its combination with an auditory rhythm, for which the mechanisms are well known. Further insights may be gained if future studies can develop methods to directly examine the perceived visual meter alone in naturalistic movement stimuli.

Linking the present proposal to the framework of musical embodiment (Leman, 2008), the proposed visual meter perception is also consistent with the earlier analyses of Charleston dancers' repetitive gestures (Leman \& Naveda, 2010; Naveda \& Leman, 2010), showing that musical meter can be mapped onto dancers' movements in space, with the spatiotemporal pattern of the feet corresponding to the 4-beat count. Whereas analyzing movements of dancers (Leman \& Naveda, 2010; Naveda \& Leman, 2010) or novices (Burger et al., 2014; Toiviainen et al., 2010) reveals how musical rhythm is embedded in the human dance, i.e., how perception is mapped onto action, the present study as well as the preceding ones (Su, 2016a; 2016b) demonstrate how action information is transformed into perception. Namely, the musical rhythm embodied in a dance movement can be extracted by visual observation. This process may be perpetuated by internal motor simulation (Jeannerod, 2001; Patel \& Iversen, 2014) that generates signals of temporal patterns, e.g., through neuronal spikes. The temporal patterns can be shared between perception (across different modalities) and action, such that perception and action can inform each other mutually. The common temporal representation also renders further cross-modal interactions possible. The degree of simulation could depend on the observer's sensorimotor experience (Jola, Abedian-Amiri, Kuppuswamy, Pollick, \& Grosbras, 2012; Kirsch \& Cross, 2015) and music or dance expertise (Karpati, Giacosa, Foster, Penhune, \& Hyde, 2016), amongst other factors. In addition, just as metrical clarity differs between different musical rhythms (Grube \& Griffiths, 2009), which accordingly influences the rhythm-to-movement mapping (Burger et al., 2014), different dance movements contain gestures and patterns of various degrees of complexity (Leman \& Naveda, 2010), not all of which communicate equal metrical strength by observation. Given the principles identified here using structured movements, the present research provides a starting point for further investigations into mechanisms involved in processing more variable dance sequences, as well as their interaction with different auditory rhythms. Along this line, more suitable auditory rhythm stimuli than the present ones may yet be designed to discriminate between the effects of different visual dance meters.

In conclusion, the present study demonstrates that the meter of dance rhythm can be visually perceived in a comparable manner to the auditory musical meter, whereby metrical grouping depends on the spatiotemporal pattern of recurrent leg movements. When watching dance movements and listening to musical rhythm simultaneously, humans form rhythmic percepts in both modalities that enable them to judge the metrical compatibility between the two. In some cases, the observed movement may even modulate auditory metrical perception. Besides likening rhythm perception in dance to that in music, the present research also compliments theories of musical embodiment by 
showing possible covert motor effects through action observation. Future research could address issues such as the metrical strength of different movements as visual stimuli, their interactions with other auditory sensorimotor processes, as well as the expertise effect. Also interesting would be the relation between the present effects (i.e., covert action to perception) and measurements of explicitly moving to music (i.e., perception to overt action), which would bridge the two aspects of actionperception coupling in a musical context.

\section{Conflict of Interest}

The author declares that the research was conducted in the absence of any commercial or financial relationships that could be construed as a potential conflict of interest.

\section{Author Contributions}

Author Y.-H. S. conceptualized and designed the study, prepared the stimuli and programmed the experiments, conducted the experiments (data collection carried out by the research assistants), analyzed the data, interpreted the results, and wrote the manuscript.

\section{$7 \quad$ Funding}

This work and the author were supported by a grant from the German Research Foundation (DFG), SU 782/1-2.

\section{Acknowledgments}

The author thanks Theresa Neumayer and Anika Berg for data collection.

\section{Reference}

Brainard, D. H. (1997). The psychophysics toolbox. Spatial Vision, 10(4), 433-436.

Burger, B., Thompson, M. R., Luck, G., Saarikallio, S. H., \& Toiviainen, P. (2014). Hunting for the beat in the body: on period and phase locking in music-induced movement. Frontiers in Human Neuroscience, 8, 1-16. http://doi.org/10.3389/fnhum.2014.00903

Cooper, G., \& Meyer, L. B. (1963). The rhythmic structure of music. (G. Cooper \& L. B. Meyer, Eds.). University Of Chicago Press.

Cross, E. S., Kraemer, D. J. M., Hamilton, A. F. de C., Kelley, W. M., \& Grafton, S. T. (2009). Sensitivity of the action observation network to physical and observational learning. Cerebral Cortex (New York, NY : 1991), 19(2), 315-326. http://doi.org/10.1093/cercor/bhn083

Doehrmann, O., \& Naumer, M. J. (2008). Semantics and the multisensory brain: How meaning modulates processes of audio-visual integration. Brain Research, 1242(C), 136-150. http://doi.org/10.1016/j.brainres.2008.03.071

Ghazanfar, A. A., \& Takahashi, D. Y. (2014). The evolution of speech: vision, rhythm, cooperation. Topics in Cognitive Science, 18(10), 543-553. http://dx.doi.org/10.1016/j.tics.2014.06.004

Grahn, J. A. (2012). See what I hear? Beat perception in auditory and visual rhythms. Experimental Brain Research, 220(1), 51-61. http://dx.doi.org/10.1007/s00221- 012-3114-8. 
Grahn, J. A. (2012). Neural Mechanisms of Rhythm Perception: Current Findings and Future Perspectives. Topics in Cognitive Science, 4(4), 585-606. http://doi.org/10.1111/j.17568765.2012.01213.x

Grube, M., \& Griffiths, T. D. (2009). Metricality-enhanced temporal encoding and the subjective perception of rhythmic sequences. Cortex, 45(1), 72-79. http://doi.org/10.1016/j.cortex.2008.01.006

Hove, M. J., Iversen, J. R., Zhang, A., and Repp, B. H. (2013a). Synchronization with competing visual and auditory rhythms: bouncing ball meets metronome. Psychological Research, 77, 388398. doi: 10.1007/s00426-012-0441-0

Hove, M. J., Fairhurst, M. T., Kotz, S. A., \& Keller, P. E. (2013b). Synchronizing with auditory and visual rhythms: An fMRI assessment of modality differences and modality appropriateness. NeuroImage, 67, 313-321. http://doi.org/10.1016/j.neuroimage.2012.11.032

Iversen, J. R., Patel, A. D., Nicodemus, B., \& Emmorey, K. (2015). Synchronization to auditory and visual rhythms in hearing and deaf individuals. Cognition 134, 232-244. doi: 10.1016/j.cognition.2014. 10.018

Jeannerod, M. (2001). Neural Simulation of Action: A Unifying Mechanism for Motor Cognition. NeuroImage, 14(1), S103-S109. http://doi.org/10.1006/nimg.2001.0832

Johansson, G. (1973). Visual perception of biological motion and a model for its analysis. Perception \& Psychophysics, 14(2), 201-211. http://doi.org/10.3758/BF03212378

Jola, C., Abedian-Amiri, A., Kuppuswamy, A., Pollick, F. E., \& Grosbras, M.-H. (2012). Motor Simulation without Motor Expertise: Enhanced Corticospinal Excitability in Visually Experienced Dance Spectators. PLoS ONE, 7(3), e33343. http://doi.org/10.1371/journal.pone.0033343.s003

Jola, C., McAleer, P., Grosbras, M.-H. L. N., Love, S. A., Morison, G., \& Pollick, F. E. (2013). Uniand multisensory brain areas are synchronised across spectators when watching unedited dance recordings. I-Perception, 4(4), 265-284. http://doi.org/10.1068/i0536

Karpati, F. J., Giacosa, C., Foster, N. E. V., Penhune, V. B., \& Hyde, K. L. (2016). Sensorimotor integration is enhanced in dancers and musicians. Experimental Brain Research, 234(3), 893903. http://doi.org/10.1007/s00221-015-4524-1

Kirsch, L. P., \& Cross, E. S. (2015). Additive Routes to Action Learning: Layering Experience Shapes Engagement of the Action Observation Network. Cerebral Cortex, 25(12), 4799-4811. http://doi.org/10.1093/cercor/bhv167

Leman, M. (2008). Embodied Music Cognition and Mediation Technology. The MIT Press.

Leman, M., \& Naveda, L. (2010). Gestures as Spatiotemporal Reference Frames for Repetitive Dance/Music Patterns in Samba and Charleston. Music Perception, 28(1), 71-91.

London, J. (2012). Hearing in Time: psychological aspects of musical meter (2nd ed.). New York: Oxford University Press. 
Maes, P.-J., Leman, M., Palmer, C., \& Wanderley, M. M. (2014). Action-based effects on music perception. Frontiers in Psychology, 4, 1-14. http://doi.org/10.3389/fpsyg.2013.01008

Manning, F., \& Schutz, M. (2013). "Moving to the beat" improves timing perception. Psychonomic Bulletin \& Review, 20(6), 1133-1139. http://doi.org/10.3758/s13423-013-0439-7

Masson, M. E., \& Loftus, G. R. (2003). Using Confidence Intervals for Graphically Based Data Interpretation. Canadian Journal of Experimental Psychology, 3, 203-220.

McGurk, H., \& MacDonald, J. (1976). Hearing lips and seeing voices. Nature 264, 746-748. doi: $10.1038 / 264746 a 0$.

Morillon, B., Hackett, T. A., Kajikawa, Y., \& Schroeder, C. E. (2015). Predictive motor control of sensory dynamics in auditory active sensing. Current Opinion in Neurobiology, 31, 230-238. http://doi.org/10.1016/j.conb.2014.12.005

Naveda, L., \& Leman, M. (2010). The Spatiotemporal Representation of Dance and Music Gestures using Topological Gesture Analysis (TGA). Music Perception, 28(1), 93-111. http://doi.org/10.1525/mp.2010.28.1.93

Patel, A. D., \& Iversen, J. R. (2014). The evolutionary neuroscience of musical beat perception: the Action Simulation for Auditory Prediction (ASAP) hypothesis. Frontiers in Systems Neuroscience, 8, 1-14. http://doi.org/10.3389/fnsys.2014.00057

Phillips-Silver, J., \& Trainor, L. (2005). Feeling the beat: Movement influences infant rhythm perception. Science (New York, NY), 308, 1-1.

Phillips-Silver, J., \& Trainor, L. J. (2007). Hearing what the body feels: auditory encoding of rhythmic movement. Cognition, 105(3), 533-546. http://doi.org/10.1016/j.cognition.2006.11.006

Phillips-Silver, J., \& Trainor, L. J. (2008). Vestibular influence on auditory metrical interpretation. Brain and Cognition, 67, 94-102.

Prinz, W. (1997). Perception and Action Planning. European Journal of Cognitive Psychology, 9(2), $129-154$.

Repp, B. H., \& Su, Y.-H. (2013). Sensorimotor synchronization: A review of recent research (20062012). Psychonomic Bulletin \& Review, 20(3), 403-452. http://doi.org/10.3758/s13423-0120371-2

Rouder, J. N., Morey, R. D., Speckman, P. L., \& Province, J. M. (2012). Default Bayes factors for ANOVA designs. Journal of Mathematical Psychology, 56(5), 356-374. http://doi.org/10.1016/j.jmp.2012.08.001

Stupacher, J., Hove, M. J., Novembre, G., Schütz-Bosbach, S., \& Keller, P. E. (2013). Musical groove modulates motor cortex excitability: A TMS investigation. Brain and Cognition, 82(2), 127-136. http://doi.org/10.1016/j.bandc.2013.03.003

Su, Y.-H. (2014). Visual enhancement of auditory beat perception across auditory interference levels. Brain and Cognition, 90(C), 19-31. http://doi.org/10.1016/j.bandc.2014.05.003 
Su, Y.-H. (2016a). Sensorimotor Synchronization with Different Metrical Levels of Point-Light Dance Movements. Frontiers in Human Neuroscience, 10, 186. http://doi.org/10.1525/mp.2011.29.1.65

Su, Y.-H. (2016b). Visual tuning and metrical perception of realistic point-light dance movements. Scientific Reports, 1-12. http://doi.org/10.1038/srep22774

Su, Y.-H., \& Pöppel, E. (2012). Body movement enhances the extraction of temporal structures in auditory sequences. Psychological Research, 76(3), 373-382. http://doi.org/10.1007/s00426011-0346-3

Su, Y.-H., \& Salazar-Lopez, E. (2016). Visual Timing of Structured Dance Movements Resembles Auditory Rhythm Perception. Neural Plasticity, 2016, 1-17. http://doi.org/10.1007/s00221-0112835-4

Toiviainen, P., Luck, G., \& Thompson, M. R. (2010). Embodied Meter: Hierarchical Eigenmodes in Music-Induced Movement. Music Perception, 28(1), 59-70. http://doi.org/10.1525/mp.2010.28.1.59

Vogt, S., Di Rienzo, F., Collet, C., Collins, A., \& Guillot, A. (2013). Multiple roles of motor imagery during action observation. Frontiers in Human Neuroscience, 7, 1-13. http://doi.org/10.3389/fnhum.2013.00807

\section{Figure legends}

Figure 1. Auditory and visual stimuli in Experiment 1. Participants watched a PLF dancing either in a duple meter $\left(1^{\text {st }}\right.$ panel $)$ or in a triple meter $\left(2^{\text {nd }}\right.$ panel $)$ to an auditory rhythm that was one of the three: metrically ambiguous ( $3^{\text {rd }}$ panel), accented in a duple meter ( $3^{\text {rd }}$ and $4^{\text {th }}$ panels $)$, or accented in a triple meter $\left(3^{\text {rd }}\right.$ and $5^{\text {th }}$ panel). The task was to judge whether the pattern of the auditory rhythm and that of the dance matched. Numbers below each visual dance sequence indicate the beat count derived from the leg movement pattern. Vertical lines and ovals in the ambiguous auditory rhythm depict discrete events of snare drum and high-hat sounds, respectively. Metrically accented rhythms consisted of the metrically ambiguous one with additional accents of a bass drum sound depicted by the arrows. Both the auditory and visual rhythms presented in the experiment lasted twice as long as depicted here (i.e., 24 IOIs in total).

Figure 2. Results of Experiment 1. (a) Mean percentages of "match" response for each visual meter condition as a function of the auditory meter, for each audiovisual tempo separately. $A$ amb, $A$ duple, and $A$ triple on the $X$-axis represent auditory conditions of metrically ambiguous, duple meter, and triple meter. Error bars represent standard error of the means. (b) Effects of visual meter contrasted at each auditory meter condition, showing the median difference scores across participants (red horizontal lines) and their 95\% CIs (the blue notches). The whiskers represent $99 \%$ of the data distribution.

Figure 3. Stimuli and procedure in Experiment 2. Participants listened to a metrically ambiguous rhythm $\left(1^{\text {st }}\right.$ panel, termed "standard rhythm") while watching a PLF dance either in a duple meter $\left(2^{\text {nd }}\right.$ panel) or in a triple meter ( $3^{\text {rd }}$ panel) to this rhythm. Following that, they heard two comparison rhythms in succession, one of which was accented in a duple meter (lower left panel) and the other in a triple meter (lower right panel). The task was to judge which of the two comparisons was more 
similar to the standard rhythm. The standard rhythm and the accompanying visual dance presented in the experiment lasted twice as long as depicted here (i.e., 24 IOIs).

Figure 4. Results of Experiment 2. Mean percentages of choosing the auditory duple-meter rhythms for each visual meter condition, at each audiovisual tempo. Error bars represent standard error of the means. 